\title{
Compromiso ganglionar extracapsular en pacientes con cáncer gástrico: determinación del valor pronóstico
}

\author{
OSCAR TAPIA E..$^{1,2}$, MIGUEL VILLASECA $\mathrm{H}^{1,2}$, \\ JUAN CARLOS ARAYA O. ${ }^{1,2}$, JUAN CARLOS ROA S., ${ }^{1,2}$
}

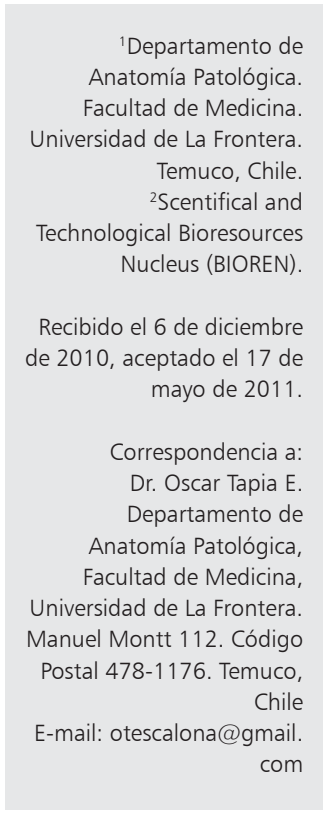

\section{Prognostic value of extracapsular lymph node involvement among patients with gastric cancer}

Background: Extracapsular lymph node involvement has a negative prognosis in malignant tumors. Aim: To assess the prognostic importance of extracapsular lymph node involvement in patients with gastric cancer with lymph node metastases. Material and Methods: Clinical and morphological features and survival of patients with gastric cancer and lymph node involvement operated between 1986 and 2003, were analyzed. Patients with and without extracapsular involvement were compared. Results: During the study period, 459 gastrectomies were performed, 312 patients (68\%) had lymph node involvement and 144 (31\%) had extracapsular involvement. Patients with and without extracapsular involvement were followed for a median of 10 (range 1 to 120) and 41 (range 1 to 193) months, respectively. Five years actuarial survival for patients with and without extracapsular involvement was 23 and $40 \%$ respectively. Extracapsular lymph node involvement and level of wall infiltration were identified as prognostic factors using a multivariate analysis. Conclusions: Extracapsular lymph node involvement is an independent risk factor for mortality among patients with gastric cancer.

(Rev Med Chile 2011; 139: 992-997).

Key words: Lymph nodes; Prognosis; Stomach neoplasms.
$\mathrm{E}$ l estadio ganglionar linfático $(\mathrm{pN})$ es uno de los principales determinantes en el pronóstico de pacientes operados por cáncer gástrico $(\mathrm{CG})^{1-5}$. La incorporación de esta variable en las distintas clasificaciones vigentes presenta diversas e importantes variaciones, mientras la clasificación Japonesa para CG (JCGC) considera para su categorización el compromiso ganglionar de acuerdo a la localización del tumor; la clasificación de la Union for International Cancer Control (UICC) y American Joint Committe on Cancer (AJCC) tan sólo consideran el número absoluto de ganglios linfáticos comprometidos independiente de la localización tumoral, agrupándolos según la última edición TNM de la AJCC para CG en N0 (sin compromiso ganglionar), N1 (1-2 ganglios comprometidos), N2 (3-6 ganglios comprometidos) y
N3 (3a: 7-15 ganglios comprometidos y 3b: >15 ganglios comprometidos); siendo esta última clasificación la más ampliamente utilizada y aceptada mundialmente dado su mejor reproducibilidad y estandarización del procesamiento histopatológico respecto a los grupos ganglionares ${ }^{6-9}$.

$\mathrm{Si}$ bien el pN basado en las clasificaciones anteriormente mencionadas son las de mayor aceptación internacional, algunos autores hacen mención a otros elementos del examen anatomopatológico de los ganglios linfáticos no incluidos en dichas clasificaciones y que presentarían valor pronóstico, tales como la proporción de ganglios linfáticos comprometidos, el tamaño de la metástasis ganglionar mayor o la presencia de extensión ganglionar extracapsular ${ }^{10-15}$.

La extensión extracapsular o periganglionar 
Valor pronóstico del compromiso ganglionar en cáncer gástrico- O. Tapia et al

ha demostrado claramente su valor pronóstico en algunos tumores como cáncer de mama, esófago, colon y laringe entre otros; siendo por tanto, informado de rutina en el reporte anatomo-patológico. En CG si bien los estudios existentes al respecto han demostrado su impacto pronóstico en la supervivencia y recurrencia de la enfermedad, estos no son numerosos y no existe consenso en la importancia del reporte de rutina en el informe anatomo-patológico. Por otro lado, y a pesar de la elevada frecuencia de esta enfermedad en nuestro país, no existen trabajos nacionales publicados que analicen su valor pronóstico ${ }^{12-14,16-25}$.

El objetivo de este estudio fue evaluar el impacto pronóstico de la extensión ganglionar extracapsular en pacientes operados por CG con metástasis ganglionares $(\mathrm{N}+)$.

\section{Material y Método}

Diseño del estudio: Estudio de cohorte prospectiva.

Marco: Se recogieron los datos provenientes de pacientes diagnosticados de CG en la Unidad de Anatomía Patológica del Hospital Hernán Henríquez Aravena de la ciudad de Temuco entre enero de 1986 y diciembre de 2003.

Participantes: Se incluyeron todos los casos de gastrectomías por CG diagnosticados de forma consecutiva en el período e institución antes señalados, en los cuales se realizó únicamente tratamiento quirúrgico con disección ganglionar extendida (D2) y cuyo recuento ganglionar fue igual o superior a 10. El seguimiento de los casos se realizó mediante el estudio de las historias clínicas y certificados de defunción obtenidos del Registro Civil e Identificación.

Criterios de exclusión: Fueron excluidos aquellos pacientes con CG sin metástasis ganglionares, sujetos sin indicación quirúrgica por enfermedad avanzada o condiciones clínicas del paciente, casos sometidos sólo a laparotomía exploradora por enfermedad diseminada, pacientes sometidos a cirugías paliativas y casos que recibieron tratamientos adicionales a la cirugía. Se excluyeron del análisis de supervivencia (SV) pacientes que fallecieron en los 30 días posteriores a la cirugía, considerados como mortalidad perioperatoria.

Protocolo de procesamiento: En este período, el procesamiento de las piezas quirúrgicas de gastrectomía fue realizado en forma uniforme a través de la apertura, extensión y fijación de la pieza quirúrgica en planchas de parafina sólida, con un examen macroscópico diferido. El examen microscópico consideró el estudio del tumor, ganglios linfáticos y ambos bordes de sección quirúrgicos (proximal y distal). Tumores avanzados fueron examinados mediante múltiples secciones representativas mientras que lesiones incipientes fueron muestreadas en su totalidad con el objeto de determinar el máximo nivel de infiltración en la pared gástrica.

Examen ganglionar linfático: La búsqueda de ganglios linfáticos fue realizada con la muestra en fresco, identificándose los respectivos grupos ganglionares y posterior fijación en formalina. Todos lo ganglios linfáticos fueron seccionados en su eje mayor, tomándose una muestra representativa y teñidos con hematoxilina-eosina. La extensión extracapsular fue definida como la infiltración de la cápsula ganglionar por células tumorales o presencia de estas en el tejido adiposo periganglionar; para ello se examinó en forma prospectiva la totalidad de láminas histológicas en el período e institución antes señalado evaluando el compromiso linfonodal y extensión extracapsular.

Variables de interés: De acuerdo a la presencia de compromiso extracapsular se obtuvieron 2 grupos: 1) Pacientes con metástasis ganglionar y sin compromiso extracapsular ( $\mathrm{pN}+\mathrm{E}-$ ) y 2) $\mathrm{Pa}$ cientes con metástasis ganglionar y compromiso extracapsular $(\mathrm{pN}+\mathrm{E}+)$. La variable resultado fue $\mathrm{SV}$, medida en meses posterior a la cirugía.

Plan de análisis: Utilizando los paquetes estadísticos Epi-info 6.0 y Stata 9.0, se realizó un análisis exploratorio de los datos, lo que determinó la necesidad de categorizar algunas variables continuas según su distribución: tamaño tumoral (menores de $40 \mathrm{~mm}$ e igual o mayores de $40 \mathrm{~mm}$ ) considerando para su categorización el eje longitudinal mayor, localización tumoral (categorizada en tres tercios: superior [cardial y subcardial], medio [cuerpo] e inferior [antral y pilórico)] acorde con 
las normas de la sociedad japonesa (en aquellos casos de tumores con compromiso de más de un segmento se categorizaron como difusos), tipo macroscópico (categorizada según la clasificación de la sociedad japonesa de gastroenterología endoscópica para tumores incipientes y según la clasificación de Bormann para tumores avanzados), nivel de infiltración (categorizado según el estadio $\mathrm{pT}$ de la $7^{\circ}$ edición de la $\mathrm{AJCC}^{7}$ para CG), grado de diferenciación histológico (categorizado en bien, moderado y poco diferenciado), prevaleciendo para su categorización el menor grado de diferenciación presente en el tumor, tipo de resección quirúrgica (gastrectomía subtotal y total), estadio ganglionar linfático y TNM de acuerdo a la $7^{\circ}$ edición AJCC para $\mathrm{CG}^{7}$. El tipo histológico de tumores según Lauren fueron clasificados en base a las definiciones ya establecidas en la literatura.

Tamaño de la muestra: No se realizó estimación de tamaño de la muestra pues se trabajó con la totalidad del universo de pacientes con CG sometidos a gastrectomía con recuento ganglionar linfático $\geq$ 10 , en el período e institución antes señalados.

Métodos estadísticos: Se aplicó estadística descriptiva con cálculo de medidas de tendencia central y extrema; y estadística analíticas, aplicando $\chi^{2}$ de Pearson y exacto de Fisher para comparación de variables categóricas; T-student para comparación de promedios en variables continuas; confección de curvas de SV de Kaplan-Meier y ulterior comparación de estas por medio de la aplicación de Log-rank test. Finalmente, se realizaron modelos de regresión logística para determinación de fuerza de asociación con cálculo de odds ratio (OR) y sus respectivos intervalos de confianza de 95\% (IC 95\%).
Tabla 1. Distribución de variables clínico-morfológicas según estado ganglionar linfático y compromiso extracapsular

\begin{tabular}{|c|c|c|c|}
\hline Variables & $\begin{array}{l}\text { CG N+E- } \\
(n=168)\end{array}$ & $\begin{array}{l}\text { CG N+E+ } \\
(n=144)\end{array}$ & $\mathbf{p}$ \\
\hline Género \% (n) & & & 0,32 \\
\hline Femenino & $32,0(54)$ & $28,0(40)$ & \\
\hline Masculino & $68,0(114)$ & $72,0(104)$ & \\
\hline Resección quirúrgica \% (n) & & & 0,35 \\
\hline Gastrectomía subtotal & $47,0(79)$ & $47,0(68)$ & \\
\hline Gastrectomía total & $53,0(89)$ & $53,0(76)$ & \\
\hline Localización \% (n) & & & 0,51 \\
\hline $1 / 3$ superior & $30,0(51)$ & $30,0(43)$ & \\
\hline $1 / 3$ medio & $23,0(38)$ & $26,0(37)$ & \\
\hline $1 / 3$ inferior & $45,0(76)$ & $40,0(58)$ & \\
\hline Difuso & $2,0(3)$ & $4,0(6)$ & \\
\hline Nivel de infiltración \% (n) & & & 0,14 \\
\hline CG incipiente & $4,0(7)$ & $1,0(2)$ & \\
\hline CG avanzado & $96,0(161)$ & $99,0(142)$ & \\
\hline Tamaño tumoral \% (n) & & & 0,65 \\
\hline$<40 \mathrm{~mm}$ & $18,0(30)$ & $16,0(23)$ & \\
\hline$\geq 40 \mathrm{~mm}$ & $82,0(138)$ & $84,0(121)$ & \\
\hline Grado de diferenciación \% (n) & & & 0,48 \\
\hline Bien diferenciado & $3,0(5)$ & $1,0(1)$ & \\
\hline Moderadamente diferenciado & $33,0(55)$ & $31,0(45)$ & \\
\hline Poco diferenciado & $64,0(108)$ & $68,0(98)$ & \\
\hline Tipo histológico según Lauren \% (n) & & & 0,11 \\
\hline Intestinal & $34,0(57)$ & $26,0(37)$ & \\
\hline Difuso & $66,0(111)$ & $74,0(107)$ & \\
\hline Estadio pT \% (n) & & & 0,93 \\
\hline pT1-pT2 & $10,0(17)$ & $10,0(15)$ & \\
\hline pT3-pT4 & $90,0(151)$ & $90,0(129)$ & \\
\hline Estadio pN \% (n) & & & 0,09 \\
\hline NO & - & - & \\
\hline N1 & $26,0(44)$ & $17,0(25)$ & \\
\hline N2 & $30,0(50)$ & $28,0(40)$ & \\
\hline N3 & $44,0(74)$ & $55,0(79)$ & \\
\hline Estadio TNM \% (n) & & & 0,02 \\
\hline I & $1,0(2)$ & $1,0(1)$ & \\
\hline$\|$ & $21,0(35)$ & $12,0(18)$ & \\
\hline III & $74,0(124)$ & $75,0(108)$ & \\
\hline IV & $4,0(7)$ & $12,0(17)$ & \\
\hline
\end{tabular}




\section{Resultados}

En el período antes señalado se realizaron 459 gastrectomías por CG correspondiendo el $68 \%$ (312) a casos con metástasis ganglionares. Se observó compromiso extracapsular en $31 \%$ (144) de los casos estudiados, siendo el promedio de ganglios linfáticos resecados 31,3 y 34,7 para tumores $\mathrm{N}+\mathrm{E}-\mathrm{y} \mathrm{N}+\mathrm{E}+$ respectivamente $(\mathrm{p}=\mathrm{NS})$; verificándose un promedio de ganglios linfáticos comprometidos por tumor para los grupos N+E- y N+E+ de 8,04 y 11,62 respectivamente $(\mathrm{p}=\mathrm{NS})$. El promedio y mediana de edad para CG N+E- fue 59,2 $\pm 11,8$ y 60 años mientras que para $\mathrm{N}+\mathrm{E}+\mathrm{de} 60,8 \pm 12,9$ y 63 años respectivamente. La distribución de variables clínico-morfológicas para cada uno de los grupos se resume en la Tabla 1, observándose entre ambos grupos estudiados diferencias esta- dísticas significativas sólo para el estadio TNM ( $\mathrm{p}$ $=0,02)$; con un mayor número de casos estadio IV en CG N+E+.

La mediana y promedio de seguimiento para CG N+E- fue de 41 (4 a 194) y 55,8 meses; para CG N+E+ esta fue de 10 (1 a 120) y 20,7 meses $(p<0,0001)$ con una SV global actuarial observada a 5 años de $44 \%$ y $23 \%$ para tumores N+E- y $\mathrm{N}+\mathrm{E}+$ respectivamente $(\mathrm{p}<0,0001)$ (Figura 1$)$. La presencia de extensión extracapsular para cada uno de los grupos según compromiso ganglionar mostró diferencias estadísticamente significativas para el grupo pN1 y pN2 (Figura 1).

El análisis multivariado (Tabla 2) permitió verificar que el nivel de infiltración parietal y el compromiso tumoral ganglionar extracapsular son variables pronósticas independientes en pacientes con CG $\mathrm{N}+$, esta última con OR de 2,7 (IC $95 \%$ de 2-3,7) y un valor de $\mathrm{p}<0,001$.

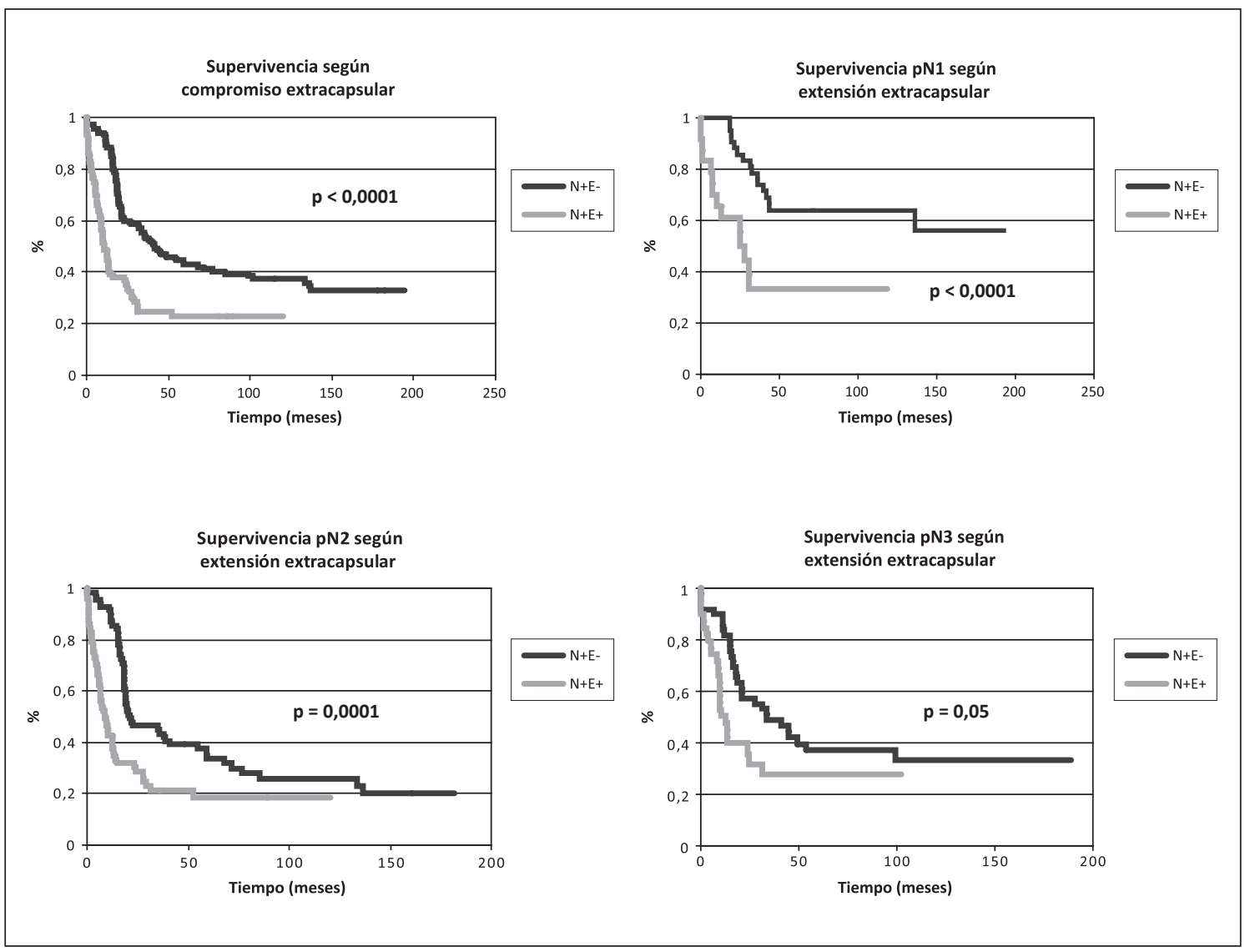

Figura 1. Supervivencia en pacientes con CG según extensión extracapsular y estadio pN. 
Tabla 2. Análisis multivariado

\begin{tabular}{|lllc|}
\hline Variable & OR & IC $\mathbf{9 5 \%}$ & p \\
\hline Gastrectomía subtotal & 1,3 & $0,9-1,9$ & 0,07 \\
\hline Tamaño $\geq 40 \mathrm{~mm}$ & 0,7 & $0,4-1,0$ & 0,11 \\
\hline Tipo difuso de Lauren & 0,9 & $0,2-3,4$ & 0,88 \\
\hline Tumor poco diferenciado & 1,4 & $0,4-4,7$ & 0,57 \\
\hline Extensión ganglionar extracapsular & 2,7 & $2,0-3,7$ & $<0,001$ \\
\hline Tumor pT3-pT4 & 1,7 & $0,9-3,8$ & 0,04 \\
\hline
\end{tabular}

\section{Discusión}

El compromiso ganglionar linfático es uno de los más importantes factores pronósticos independientes en pacientes con $\mathrm{CG}^{1-5}$. El actual sistema de estadificación ganglionar basado en la $7^{\circ}$ edición de la AJCC sólo considera el número absoluto de ganglios linfáticos comprometidos por tumor para su categorización, sin hacer mención ni considerar para ello características de este compromiso nodal, como tamaño de la metástasis ganglionar o extensión tumoral extracapsular; entendiendo por este último como la infiltración tumoral de la cápsula ganglionar o presencia de células neoplásicas en el tejido adiposo periganglionar ${ }^{7}$.

Si bien en otros tumores malignos ${ }^{14,16-19,21,22}$ (cabeza y cuello, mama, recto, vulva, vejiga, próstata y esófago entre otros) esta claramente demostrado el impacto pronóstico de la extensión extracapsular en pacientes con metástasis nodal; los estudios al respecto para cáncer gástrico son escasos, demostrándose, sin embargo, en ellos el valor pronóstico negativo del compromiso extracapsular relacionado tanto con diseminación tumoral peritoneal, metástasis hepáticas y tasa de supervivencia significativamente menores comparados con sujetos que presentan metástasis linfáticas sin extensión extracapsular ${ }^{12-14,20,23-25}$.

En nuestro estudio verificamos en el análisis bivariado que pacientes con compromiso extracapsular presentan supervivencias significativamente menores que sujetos sin extensión extracapsular $(\mathrm{p}<0,0001)$, del mismo modo, el análisis multivariado permitió demostrar que el compromiso tumoral ganglionar extracapsular en pacientes con CG N+ es un factor pronóstico independiente, presentando estos sujetos un riesgo 2,7 veces mayor de fallecer que aquellos pacientes que no lo presentan ( $\mathrm{p}<0,001$; IC 95\% 2-3,7). Es importante mencionar que el análisis por grupos separados, demostró para tumores pN1 y pN2 el valor pronóstico de esta variable, mientras que para tumores $\mathrm{pN} 3$ no se observaron diferencias estadísticas significativas $(p=0,05)$, estando en este grupo el pronóstico probablemente determinado por el número de ganglios comprometidos $(>6$ metástasis ganglionares) más que por la presencia de extensión extracapsular.

Si bien en la actualidad la clasificación TNM es la más ampliamente aceptada y utilizada por su claridad, sencillez y adecuada correlación pronóstica con recurrencia y supervivencia, la evaluación de la extensión ganglionar extracapsular no es considerada para la definición del estadio linfonodal ( $\mathrm{pN})$; y por lo tanto, no informado de rutina en el reporte anatomo-patológico, omitiéndose de esta forma información pronóstica relevante que permite obtener subgrupos de pacientes con CG $\mathrm{N}+$, fundamentalmente $\mathrm{pN} 1$ y $\mathrm{pN} 2$ que se podrían beneficiar de terapias complementarias.

En conclusión, estos resultados nos permiten validar en nuestra población de estudio el valor pronóstico independiente de la extensión tumoral ganglionar extracapsular, debiendo por tanto, esta variable informarse de rutina en el reporte anatomo-patológico.

\section{Referencias}

1. Kim DY, Seo KW, Joo JK, Park YK, Ryu SY, Kim HR, et al. Prognostic factors in patients with node-negative gastric carcinoma: a comparison with node-positive gastric carcinoma. World J Gastroenterol 2006; 12: 1182-6.

2. Noguchi M, Miyazaki I. Prognostic significance and surgical management of lymph node metastasis in gastric cancer. Br J Surg 1996; 83: 156-61. 
3. Kim JP, Lee JH, Kim SJ, Yu HJ, Yang HK. Clinicopathologic characteristics and prognostic factors in 10783 patients with gastric cancer. Gastric Cancer 1998; 1: 125-33.

4. Siewert JR, Böttcher K, Stein HJ, Roder JD. Relevant prognostic factors in gastric cancer: ten-year results of the German Gastric Cancer Study. Ann Surg 1998; 228: 449-61.

5. Roder JD, Bottcher K, Busch R, Wittekind C, Hermanek $\mathrm{P}$, Siewert JR. Classification of regional lymph node metastasis from gastric carcinoma. German Gastric Cancer Study Group. Cancer 1998; 82: 621-31.

6. Japanese Gastric Cancer A. Japanese Classification of Gastric Carcinoma - 2nd English Edition. Gastric Cancer 1998; 1:10-24.

7. Edge SB, Byrd DR, Carducci MA, Compton CC, eds. AJCC Cancer Staging Manual. 7th ed. New York, NY: Springer; 2009.

8. Ikeguchi M, Murakami D, Kanaji S, Ohro S, Maeta Y, Yamaguchi K, et al. Lymph node metastasis of gastric cancer: comparison of Union for International Cancer Control and Japanese systems. ANZ J Surg 2004; 74: 852-4.

9. Ichikura T, Tomimatsu S, Okusa Y, Uefuji K, Tamakuma S. A comparison of the prognostic significance between the number of metastatic lymph nodes and nodal stage in gastric carcinoma. Hepatogastroenterology 1999; 46: 3281-6.

10. Inoue $\mathrm{K}$, Nakane $\mathrm{Y}$, liyama $\mathrm{H}$, Sato $\mathrm{M}$, Kanbara T, Nakai $\mathrm{K}$, et al. The superiority of ratio-based lymph node staging in gastric carcinoma. Ann Surg Oncol 2002; 9: 27-34.

11. Dhar DK, Kubota H, Kinukawa N, Maruyama R, Kyriazanos ID, Ohno S, et al. Prognostic significance of metastatic lymph node size in patients with gastric cancer. Br J Surg. 2003; 90: 1522-30.

12. Alakus H, Hölscher AH, Grass G, Hartmann E, Schulte C, Drebber U, et al. Extracapsular lymph node spread: a new prognostic factor in gastric cancer. Cancer 2010; 116: 309-15.

13. Nakamura K, Ozaki N, Yamada T, Hata T, Sugimoto S, Hikino $\mathrm{H}$, et al. Evaluation of prognostic significance in extracapsular spread of lymph node metastasis in patients with gastric cancer. Surgery 2005; 137: 511-7.

14. Wind J, Lagarde SM, Ten Kate FJ, Ubbink DT, Bemelman WA, van Lanschot JJ. A systematic review on the significance of extracapsular lymph node involvement in gastrointestinal malignancies. Eur J Surg Oncol 2007; 33: 401-8.

15. Cheong O, Oh ST, Kim BS, Yook JH, Kim JH, Im JT, et al. Large metastatic lymph node size, especially more than $2 \mathrm{~cm}$ : independent predictor of poor prognosis in node-positive gastric carcinoma. World J Surg 2008; 32 : 262-6.

16. Brasilino de Carvalho M. Quantitative analysis of the extent of extracapsular invasion and its prognostic significance: a prospective study of 170 cases of carcinoma of the larynx and hypopharynx. Head Neck 1998; 20: 16-21.

17. Fleischmann A, Thalmann GN, Markwalder R, Studer UE. Prognostic implications of extracapsular extension of pelvis lymph node metastases in urothelial carcinoma of the bladder. Am J Surg Pathol 2005; 29: 89-95.

18. Griebling TL, Ozkutlu D, See WA, Cohen MB. Prognostic implications of extracapsular extension of lymph node metastases in prostate cancer. Mod Pathol 1997; 10: 804-9.

19. van der Velden J, van Lindert AC, Lammes FB, ten Kate FJ, Sie-Go DM, Oosting H, et al. Extracapsular growth of lymph node metastases in squamous cell carcinoma of the vulva. The impact on recurrence and survival. Cancer 1995; 75: 2885-90.

20. Koike H, Ichikawa D, Kitamura K, Tsuchihashi Y, Yamagishi H. Perinodal involvement of cancer cells in gastric cancer patients. Surgery 2004; 135: 266-72.

21. Mambo NC, Gallager HS. Carcinoma of the breast: the prognostic significance of extranodal extension of axillary disease. Cancer 1977; 39: 2280-5.

22. Ueno $\mathrm{H}$, Mochizuki $\mathrm{H}$. Clinical significance of extranodal skipped cancer infiltration in rectal cancer. Surgery Today 1997; 27: 617-22.

23. Tanaka T, Kumagai K, Shimizu K, Masuo K, Yamagata K. Peritoneal metastasis in gastric cancer with particular reference to lymphatic advancement; extranodal invasion is a significant risk factor for peritoneal metastasis. J Surg Oncol 2000; 75: 165-71.

24. Kumagai K, Tanaka T, Yamagata K, Yokoyama N, Shimi$\mathrm{zu}$ K. Liver metastasis in gastric cancer with particular reference to lymphatic advancement. Gastric Cancer 2001; 4: 150-5.

25. Di Giorgio A, Botti C, Sammartino P, Mingazzini P, Flammia M, Stipa V. Extracapsular lymphnode metastases in the staging and prognosis of gastric cancer. Int Surg 1991; 76: 218-21. 